\title{
Neurophysiological assessment of divers with medical histories of neurological decompression illness
}

\author{
A W Murrison, E Glasspool, R J Pethybridge, T J R Francis, E M Sedgwick
}

\begin{abstract}
Objective-To examine the possibility that subclinical damage may persist after clinical recovery from neurological decompression illness.

Methods-The neuraxes of 71 divers with medical histories of neurological decompression illness and 37 non-diver controls were examined by recording the somatosensory evoked potentials produced on stimulation of the posterior tibial and median nerves.

Results-Although the tests gave some objective support for the presence of "soft" residual neurological symptoms and signs, no evidence was given for the presence of subclinical damage.

Conclusions-The contention that neurological damage persists after full clinical recovery from the neurological decompression illness was not supported.
\end{abstract}

(Occup Environ Med 1994;51:730-734)

Keywords: divers, decompression illness, somatosensory evoked potentials

The principal finding from early postmortem studies of professional divers and compressed air workers who had died from acute neurological decompression illness was the presence in the spinal cord of scattered discrete haemorrhages of the white matter with relative sparing of grey matter. ${ }^{\star}$ In divers, the lower thoracic, upper lumbar, and lower cervical cord were preferentially involved. A similar pattern was found in the spinal cords of people who had died several months or years after sustaining neurological decompression illness with most of the damage being located in the dorsal and lateral columns. ${ }^{14-6}$ Most of these reports related to patients with substantial functional residua. Palmer et al described unexpectedly extensive damage to a spinal cord at necropsy in a patient with a medical history of neurological decompression illness who had effected a good clinical recovery before his death, which was not related to diving. ${ }^{5}$ Palmer et al suggested that neurophysiological studies might be used to supplement the physical examination of divers. Certainly

*The convention for describing the decompression disorders outlined by Elliott and Moon ${ }^{2}$ has been adopted throughout the text. Thus the generic term neurological decompression illness is used to encompass type II decompression sickness and cerebral arterial gas embolism. ${ }^{3}$ with the degree of disruption shown by Palmer et $a l^{5}$ obvious neurophysiological abnormalities would have been expected before death. Palmer et al found chronic spinal cord degeneration at postmortem examination in seven out of an uncontrolled series of three amateur and eight professional divers. ${ }^{6}$ The divers lacked documented medical histories of decompression illness and had been certified as neurologically intact at medical examinations performed shortly before death. The abnormalities consisted of gliosis, corpora amylacea, and blood vessel hyalinisation. A limited number of sections of each spinal cord were examined by the Marchi method for showing myelin degradation products. The size of the "window of opportunity" for detecting degenerate myelin is controversial but it probably starts at 10-20 days after an insult and lasts for an undefined period (months or years) thereafter. ${ }^{7}$ It showed long tract degeneration at postmortem examination in three of the commercial divers. The method is, however, prone to misinterpretation because of artefact and lack of standard preparation. $^{8}$ Subsequently, Palmer et al showed cerebral vasculopathy at postmortem examination in divers. ${ }^{9}$ This supported ill defined electroencephalographic ${ }^{10}$ and psychometric $^{11} 12$ evidence for the existence of subclinical cerebral deficits in divers and compressed air workers. It was of particular interest in the light of the findings by Polkinghorne et al of retinal vascular and pigment epithelial defects in divers and the possibility that such damage could be indicative of wider changes to the central nervous system. ${ }^{13}$

Somatosensory evoked potentials (SSEPs) are recorded over the spine and somatosensory cortex after the electrical stimulation of a mixed nerve trunk and the subsequent passage of impulses along the dorsal columns. ${ }^{14}$ They consist of positive and negative deflections that are identified according to the characteristic latency at which they follow the initiating stimulus and by their amplitude. Clinically, the SSEPs to the stimulation of the posterior tibial nerve (PTSEP) and the median nerve (MSEP) are most commonly recorded. Central conduction time may be calculated by subtracting the latency recorded over the spine from the corresponding latency recorded over the scalp. Figure 1 shows the principal parameters used to describe PTSEP and MSEP data. These were adopted as the outcome variables in this study. The P40 wave represents the primary response of the foot area of the somatosensory cortex to 
Figure 1 Somatosensory evoked potentials on stimulation of $(A)$ the posterior tibial and $(B)$ median nerves.

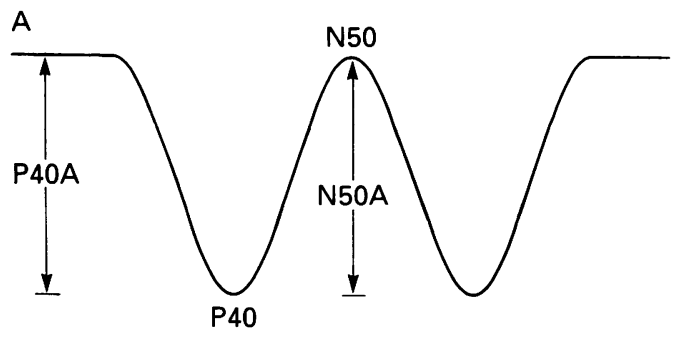

B

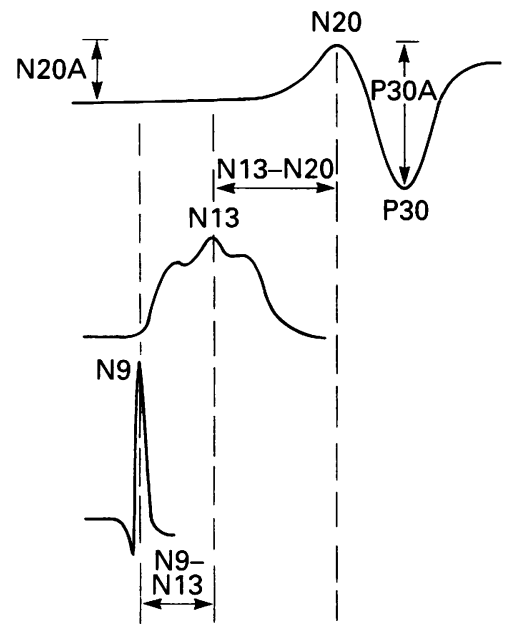

stimulation, ${ }^{15}$ and its latency (P40L) has been found to be the most reproducible feature of the PTSEP in clinical studies. ${ }^{16}$ In view of this, the P40L was adopted as the principal variate in this study. After stimulation of the median nerve, the $\mathrm{N} 20$ wave over the scalp, the N13 at the seventh cervical vertebra, and the N9 recorded above the clavicle at Erb's point are the most reliable features. From these the conduction time from Erb's point to C7 (N9-N13) and the central conduction time from $\mathrm{C} 7$ to scalp (N13-N20) may be derived.

The SSEPs are well established as a means of invasive monitoring the spinal cord function of dogs with neurological decompression illness. ${ }^{17}$ Neurophysiological studies into human neurological decompression illness have appeared only as brief reports, ${ }^{18}$ single case studies, ${ }^{1920}$ letters, ${ }^{21}$ or in the proceedings $^{22}$ or abstracts ${ }^{23}$ of meetings. All have involved small numbers of patients and in each the description of the method is limited, particularly with regard to the control data. Yiannikas and Beran compared the SSEPs of 30 non-diver controls with those of 30 divers who were within four weeks of treatment for decompression illness of unspecified nature and severity. It was found that lumbar spine to scalp central conduction times were abnor$\mathrm{mal}$ in nine of the divers of which six were clinically normal. Smith and Trojaborg found that SSEP latencies and T12 to scalp and C7 to scalp central conduction times were normal in nine out of 10 patients with ill defined acute decompression illness. ${ }^{21}$ Moon et al detected abnormalities of the PTSEP in seven out of 12 cases of acute neurological decompression illness. ${ }^{22}$ This was unremarkable as, of the 12 cases, 11 had clinical involvement of the lower limbs and 10 of these had motor deficits. None the less, the hypothesis that SSEPs may be capable of detecting subclinical spinal cord damage in divers is encouraged by their ability to show subclinical dysfunction in disorders such as multiple sclerosis, ${ }^{24}$ a disease that shares some of the multi focal characteristics of neurological decompression illness. The abnormalities of SSEPs have been shown to persist on reversion to clinical normality after the relief of spinal cord compression caused by meningioma, ${ }^{14}$ and may be more sensitive than physical examination in showing abnormalities in cervical myelopathy..$^{25}$

In the light of the findings from the postmortem examinations of divers and compressed air workers with and without histories of decompression illness, ${ }^{14-6}$ our study was designed as an in vivo examination of the possibility that residual neurological damage may persist after clinical recovery from neurological decompression illness.

\section{Materials and methods}

Potential subjects who had been treated for neurological decompression illness between one month and four years previously (mean 17 months) were identified from the records of the Institute of Naval Medicine, the Diving Diseases Research Centre, Plymouth and the hyperbaric unit of Whipps Cross Hospital, London. Four cases were referred by Health and Safety Executive approved diving physicians. Two patients in whom the diagnosis of decompression illness was questionable were excluded. ${ }^{6}$ Four potential participants with histories of other neurological illness or injury, including head injury with loss of consciousness, or serious systemic conditions were also excluded. A total of 71 divers were admitted to the study (12 professionals and 59 amateurs). Thirty seven non-diver controls were drawn from a wide range of workers at the Institute of Naval Medicine, Southampton General Hospital, Diving Diseases Research Centre, and auxiliary coastguards services. Standards for entry into the study were the same for divers and non-divers. Specific coincident neurological injuries caused individual test results to be discarded from several sets of data that were otherwise acceptable. Thus a history of low back pain would lead to the exclusion of PTSEPs but not MSEPs. This resulted in a slight disparity between the number of volunteers with admissible PTSEP data and the number with MSEP data. For PTSEP data, the mean (range) age of non-divers ( $\mathrm{n}=$ 35) was $31 \cdot 1$ (19-55) and the mean (range) height was $1.74(1.54-1.92)$ m compared with the divers $(n=61)$ age $34 \cdot 6(20-62)$ and height $1.77(1.60-1.91) \mathrm{m}$. For MSEPs data, the age of non-divers $(n=37) 31.9(19-59)$ and height $1.74(1.54-1.92) \mathrm{m}$ compared with the divers $(n=71)$ age $35.5(20-62)$ and height $1.78(1.60-1.96) \mathrm{m}$. Sex was discarded as an independent variable (three divers and six non-divers were women) as the influence of sex on SSEPs is accounted for by allowing for height. ${ }^{16}$ The divers were asked to estimate 
the number of dives that they had performed. Their estimates ranged from four to 3000 dives with a mean of 454 .

The clinical status of the divers at the time of injury was deduced by a review of the case notes and the current state of divers and nondiver controls was determined from their medical history and neurological examination, which was performed by a single physician (AWM). Each limb was assessed as having "hard" or "soft" neurological signs or symptoms at the time of participation in the study. For example, the demonstration of a definite motor weakness or a convincing area of numbness would be regarded as a hard finding, whereas a diver's report that the sensation of pin prick was altered but in whom an objective deficit could not be shown would be classified as a soft feature. Divers were assigned to subgroups according to the symptoms and signs in the worse affected upper and lower limbs. An individual could be in the "clinically normal" subgroup for the upper limbs and in the clinically abnormal subgroup for the lower limbs (hard or soft).

SSEPs were recorded with a Neuropak Four Mini (Nihon Kohden) machine in accordance with the method described by Katifi and Sedgwick. ${ }^{16}$ Recording electrodes were sited over the scalp at Cz', C3', C4', $\mathrm{FpZ},{ }^{26}$ over the seventh cervical vertebra and at both Erb's points. The SSEPs were not recorded over the lumbar spine as it was found that they tended to be obscured by muscular artifact in the predominantly muscular male population studied. Earth leads were connected to the subjects' limbs and bipolar stimulating electrodes were placed over the posterior tibial nerve at a point below and posterior to the medial malleolus, or over the median nerve at the wrist. Volunteers were monitored in the reclining position.

Summary of multivariate comparisons of divers and non-divers

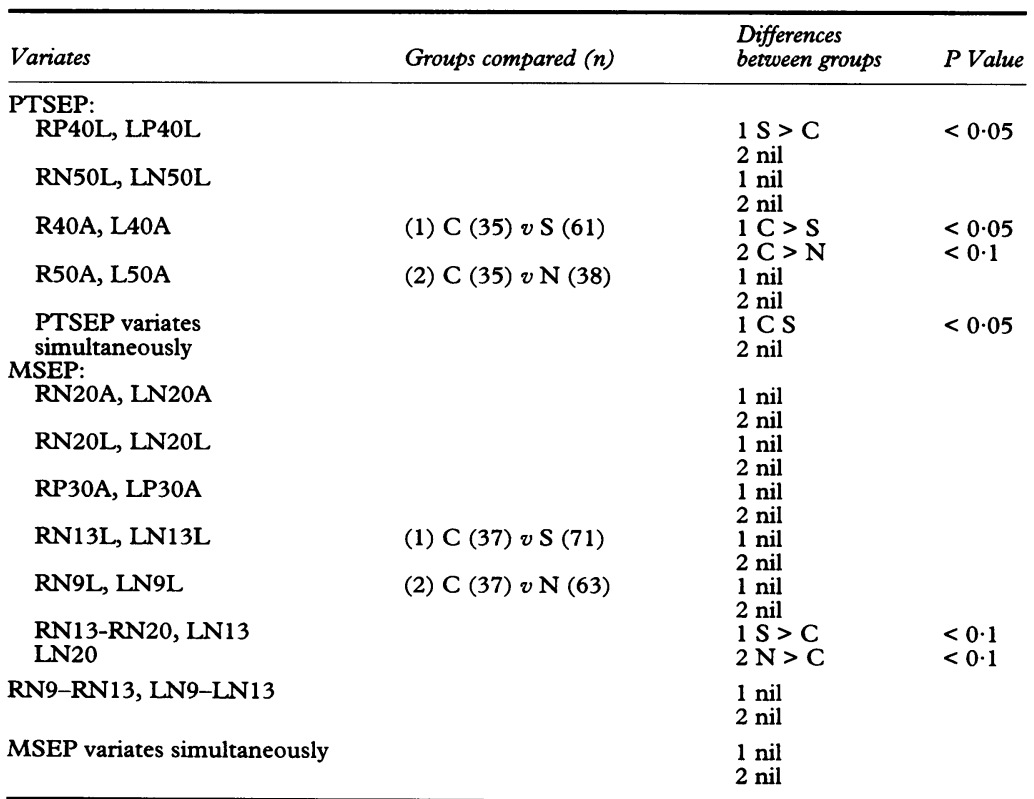

Prefix $\mathrm{R}=$ right limb; $\mathrm{L}=$ left limb; suffix $\mathrm{L}=$ latency; $\mathrm{A}=$ amplitude; $\mathrm{C}=$ non-divers; $\mathrm{S}=$ tota divers; $\mathrm{N}=$ divers with clinically normal legs or arms; PTSEP = leg data; $\mathrm{MSEP}=$ arm data.
They were asked to relax and were kept warm. The intensity of the stimulus used was that at which a definite twitch of at least one digit was seen. Two sequences of at least 500 impulses were averaged separately and summated to confirm reproducibility.

Volunteers gave their written informed consent to the procedure, which had the approval of the Ethics Committees of Southampton General Hospital and the Royal Navy.

\section{Results}

We carried out multivariate analyses of variance and covariance, with Wilk's $\lambda$ and Rao's $F$ test statistics, ${ }^{27}$ to determine any differences between groups. The analyses incorporated the data from both the right and the left side in each set of limbs.

In the non-diver control group it was found that several of the SSEP latency (suffix L) and amplitude (suffix A) variates were linearly related positively and negatively respectively to height and, to a lesser extent, to age. In view of this, it was considered necessary to compare non-divers with divers by multivariate analysis of covariance with height and age as the covariates. Where the covariates were found not to make a useful contribution to the analysis, a multivariate analysis of variance was conducted. The analysis of PTSEPs was conducted with the following as bivariates in which the prefix $\mathbf{R}$ indicates the results from stimulation of the right and $L$ the left side (fig 1A); RP40L and LP40L, RN50L and LN50L, RP40A and LP40A, and RN50A and LN50A. Similarly, in the analysis of MSEPs, the following sets of bivariates were used (fig 1B); RN20A and LN20A, RN20L and LN20L, RP30A and LP30A, RN13L and LN13L, RN9L and LN9L, RN13-RN20 and LN13-LN20, and RN9-RN13 and LN9LN13. Also, combined multivariate analyses were performed for the PTSEP and MSEP sets of variates.

Out of 61 divers with admissible PTSEP data 23 were assessed as having clinical abnormalities of the lower limbs. Eight of the 71 divers with admissible MSEP data were similarly assessed. All of the non-diver controls were normal on physical examination at the time of their participation in the study.

Of the various analyses conducted on nondivers and divers (or subgroups of divers), the only significant differences between groups were associated with P40L, P40A, N50L and the combined PTSEP variates. None of the MSEP differences reached significance (table).

PTSEP DATA

The 61 divers entered into the analysis of PTSEP data had, on average, significantly longer P40Ls than the corresponding 35 nondivers $(P<0.01$, table). The relation was strengthened by limiting the comparison to the 23 divers who had lower limb clinical abnormalities in at least one leg (hard or soft) and it persisted when the 15 divers with soft clinical abnormalities only were compared 


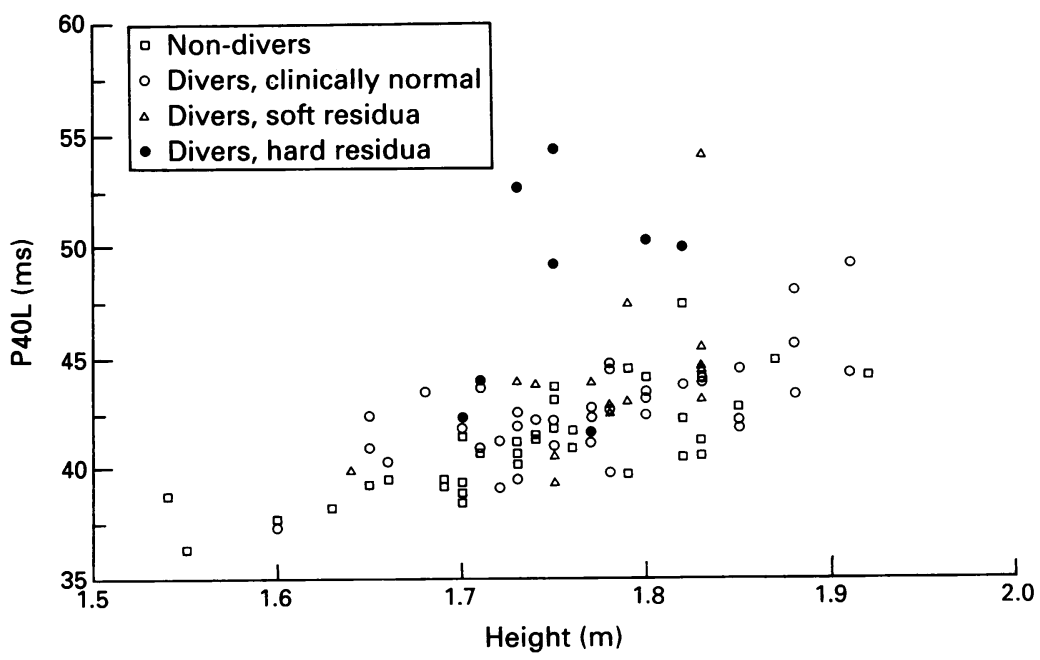

Figure 2 Relation between the average of left and right P4OLs and height in 35 nondivers and 61 divers with medical histories of neurological decompression illness.

with non-divers $(P<0.01)$. No difference was detected between divers with clinically normal lower limbs $(n=38)$ and non-divers. Figure 2 shows the separation in terms of P40L against height of non-divers, divers with hard and with soft clinical abnormalities of the lower limbs, and divers with clinically normal legs.

The only significant N50L differences between non-divers and divers were the prolonged latencies seen in the 23 divers with soft and hard abnormalities of the legs $(P<0.05)$ and the 15 divers with soft features only $(\mathrm{P}<$ 0.05).

The 61 divers had significantly smaller values for P40A than non-divers $(P<0.05)$ as did a subgroup of 23 divers with clinical abnormalities in the lower limbs (hard or soft) $(P<0.05)$. Although no significant difference in P40A was found between the non-divers and the 15 divers with soft lower limb clinical abnormalities, there was a suggestion that the 38 divers with clinically normal legs had smaller amplitude values than non-divers $(\mathbf{P}<$ $0 \cdot 1)$. There was an indication $(P<0 \cdot 1)$ that the N50A of the non-diver controls exceeded the subgroup of divers with clinical abnormalities of the lower limbs $(n=23)$.

Multivariate analysis of the combined PTSEP variates indicated that non-divers differed from divers $(P<0.05)$. There was no difference between non-divers and divers with clinically normal legs.

No significant PTSEP differences were shown between the 14 divers whose abnormalities of the lower limbs at presentation had resolved fully by the time of their participation in the study and the 35 non-diver controls. No significant difference was found between the non-divers and the 14 divers without clinical abnormalities in the lower limbs who had performed at least 250 dives and were at least six months after any accident.

\section{MSEP DATA}

The suggestion of a longer N13-N20 amongst the 71 divers with MSEP data compared with 37 non-divers (table) did not reach significance $(P<0 \cdot 1)$. This was also the case when the diver group was restricted to those whose arms were both clinically normal. No MSEP differences emerged between the non-divers and the 28 divers who were recorded as having upper limb abnormalities at presentation for treatment, but whose arms were clinically normal when monitored. No significant differences were found between the non-divers and the 23 divers with clinically normal arms who had performed at least 250 dives and who were monitored at least six months after their diving accident.

\section{Discussion}

Differences in PTSEP values were found between non-divers and divers with medical histories of neurological decompression illness particularly when restricting the analysis to divers with soft and hard or merely soft residual clinical abnormalities. No differences were shown, however, between controls and divers with clinically normal lower limbs. The MSEP variates did not show any significant differences between divers and non-divers. Thus, although SSEPs gave objective confirmation of hard and soft residua, none of the variates examined supported the contention that there exists residual, sub-clinical neurological damage in divers who have apparently fully recovered from decompression illness. These findings concur with those of Moon et $a .^{22}$ Although Yiannikas and Beran's neurophysiological work suggested that occult lesions existed in patients with neurological decompression illness, ${ }^{18}$ it is possible, from the data given, that several of the subjects studied would have been classified as soft cases in this study with consequent agreement with the findings reported here.

Limited evidence emerged against the contention that diving itself may cause neuraxial damage, particularly as those who had performed in excess of 250 dives and who had clinically normal arms or legs were indistinguishable on the ground of MSEP and PTSEP data from non-divers.

Conclusions from this study are limited by the assumption that the measurement of SSEPs is appropriate to show subtle damage in diving related illness. Although SSEPs are widely used to show subclinical changes in multiple sclerosis, this disease is characterised by demyelination. Although diving related illness involves demyelination, axonal damage and gliosis are also features. ${ }^{56}$ Furthermore, the search for very subtle changes in asymptomatic people is likely to be affected by the relatively small sample size and the limitations of the control data in this study. None the less, with what are commonly regarded as sensitive investigative tools, the degree of dorsal column disruption shown in divers at postmortem examination by Palmer et $a l^{56}$ and Calder et $a l^{4}$ was not detected neurophysiologically.

This study was funded by the Department of Energy and was supported by the Medical Director General (Navy). The assistance of Professor DH Elliott, Surgeon Commander JJW Sykes Royal Navy of the Institute of Naval Medicine, and of Dr MR Cross of the Diving Diseases Research Centre is gratefully acknowledged. 
1 Haymaker W. Decompression sickness. In: Lubarsch C, Henke F, Rossle R, eds. Handbuch der speziellen pathologischen: Anatomie und Histologie. Vol XIII. Berlin Springer-Verlag, 1957:1600-72.

2 Elliott DH, Moon RE. Manifestations of the decompression disorders. In: Bennett PB, Elliott DH, eds. The physiology and medicine of diving, 4th ed. London: WB Saunders, 1993.

3 Elliott DH, Kindwall EP. Manifestations of the decompression disorders. In: Bennett PB, Elliott DH, eds. The physiology and medicine of diving, 3rd ed. London: physiology and mediere Tindall, 1982

4 Calder IM, Palmer AC, Hughes JT, Bolt JF, Buchanan JD. Spinal cord degeneration associated with type II Jecompression sickness: case report. Paraplegia 1989; decompressi

5 Palmer AC, Calder IM, McCallum RI, Mastaglia FL Spinal cord degeneration in a case of "recovered" spinal cord decompression sickness. BMF 1981;283:888-90.

6 Palmer AC, Calder IM, Hughes JT. Spinal cord degeneration in divers. Lancet 1987; ii: $1365-7$.

7 Smith MC. Observations on the extended use of the Marchi method. I Neurol Neurosurg Psychiatry 1956; 19:67-73.

8 Strich SJ. Notes on the Marchi method for staining degenerating myelin in the peripheral and central nervous system. $f$ Neurol Neurosurg Psychiatry 1968;31: vous syst $110-4$.

9 Palmer AC, Calder IM, Yates PO. Cerebral vasculopathy in divers. Neuropathol Appl Neurobiol 1992;18:113-24.

10 Rozsahegyi I, Roth B. Participation of the central nervou system in decompression. Industrial Medicine and Surgery 1966;35:101-10

11 Peters BH, Levin HS, Kelley J. Neurologic and psychologic manifestations of decompression illness in divers. Neurology 1977;27:125-7.

12 Todnem K, Nyland H, Skeidsvoll H, Svihus R, Rinck P Riise $T$, et al. Neurological long term consequences of deep diving. $\mathrm{Br} \mathcal{F}$ Ind Med 1991;48:258-66.

13 Polkinghorne PJ, Sehmi K, Cross MR, Minassian D, Bird AC. Ocular fundus lesions in divers. Lancet 1988; ii: $1381-3$.

14 Halliday AM, Wakefield GS. Cerebral evoked potentials in patients with dissociated sensory loss. $\mathcal{F}$ Neurol Neurosurg patients with dissociated sen
Psychiatry 1963;26:211-9.

15 Seyal M, Emerson RG, Pedley TA. Spinal and early scalprecorded components of the somatosensory evoked potential following stimulation of the posterior tibial nerve. Electroenceph Clin Neurophysiol 1983;55:320-30

16 Katifi AH, Sedgwick EM. Somatosensory evoked potentials from posterior tibial nerve and lumbo-sacral dermatomes. Electroenceph Clin Neurophysiol 1986;65:49-59.

17 Leitch DR, Hallenbeck JM. Somatosensory evoked potentials and neuraxial blood flow in central nervous system decompression sickness. Brain Res 1984;311:307-15.

18 Yiannikas C, Beran R. Somatosensory evoked potentials, electroencephalography and CT scans in the assessment of the neurological sequelae of decompression sickness. Clin Exp Neurol 1989;25:91-6.

19 Synek VM, Glasgow GL. Recovery from alpha coma after decompression sickness complicated by spinal cord decompression sickness complicated by spinal cord
lesions at cervical and midthoracic levels. Electroenceph lesions at cervical and midthoracic

20 Sedgwick EM. Somatosensory evoked potentials in a case of decompression sickness. In: Long Term Health Effects Working Group of the Medical Research Council Decompression Sickness Panel, eds. Diagnostic techniques in diving neurology. London: MRC, 1987:74-6.

21 Smith T, Trojaborg W. Somatosensory evoked potentials and spinal decompression sickness. Lancet 1988;i: 364-5

22 Moon RE, Camporesi EM, Erwin CW. Use of evoked potentials during acute dysbaric illness. In: Long Term Health Effects Working Group of the Medical Research Council Decompression Sickness panel, eds. Diagnostic Council Decompression Sickness panel, eds. Diagnostic techniques in diving

23 Overlock R, Dukta A, Farm F, Okamoto G, Suzuki D. Somatosensory evoked potentials measured in divers with a history of spinal cord decompression sickness. Undersea Biomedical Research 1989;16(suppl):89.

24 Van Buggenhout E, Ketelaer P, Carton H. Success and failure of evoked potentials in detecting clinical and subclinical lesions in multiple sclerosis patients. Clin Neurol Neurosurg 1982;84:3-14.

$25 \mathrm{Yu} \mathrm{YL}$, Jones SJ. Somatosensory evoked potentials in cervical spondylosis. Correlation of median, ulnar and posterior tibial nerve responses with clinical and radiological findings. Brain 1985;108:273-300.

26 Jasper HH. The ten-twenty electrode system of the International Federation for Electroencephalography. Electroenceph Clin Neurophysiol 1958:10;371-5.

27 Morrison DF. Multivariate statistical methods. New York McGraw Hill, 1976.

\section{Correspondence and editorials}

Occupational and Environmental Medicine welcomes correspondence relating to any of the material appearing in the journal. Results from preliminary or small scale studies may also be published in the correspondence column if this seems appropriate. Letters should be not more than 500 words in length and contain a minimum of references. Tables and figures should be kept to an absolute minimum. Letters are accepted on the understanding that they may be subject to editorial revision and shortening.

The journal also publishes editorials which are normally specially commissioned. The Editor welcomes suggestions regarding suitable topics; those wishing to submit an editorial, however, should do so only after discussion with the Editor. 